Eduardo Di Mauro, Xinda Li, Christian Pellerin, Fabio Cicoira, Member, IEEE, Clara Santato, Member, IEEE

\title{
Smart Packaging in the Sustainability Challenge: Eumelanin as a UV-absorption Enhancer of Polymers
}

\begin{abstract}
The black-brown biopigment eumelanin screens the harmful ultraviolet (UV) radiation in the human skin. In industry, the absorption in the UV range of plastics is improved by the inclusion of additives. Our work reports an evaluation of the biopigment eumelanin as a nature-inspired additive for polymers to improve their UV-absorption. Eumelanin being non-toxic and produced by natural sources, it represents an interesting candidate for sustainable plastic additives. In our work, we fabricated eumelanin-including films of commercial linear low-density polyethylene (LLDPE), a plastic ubiquitous in packaging applications. The films were obtained by meltcompounding and compression molding. The Melanin Free Acid treatment (MFA) was used to enhance the biopigment dispersion in the films. We observed that amounts of eumelanin as low as $0.8 \%$ wt. bring about an increase of the UV absorption, up to one order of magnitude in the UVA range. We also assessed the effect of eumelanin on the film stability under prolonged UV irradiation: the biopigment proved to work both as UV-absorption enhancer but also as a photo-prooxidant, as inferred by thermogravimetric analysis (TGA) and infrared (IR) spectroscopy.
\end{abstract}

Index Terms - Melanin, Melanin Free Acid, Linear LowDensity Polyethylene, Packaging, UV-absorbers, UV-aging.

\section{INTRODUCTION}

$\mathrm{T}$ he packaging industry employs synthetic UV stabilizers to reduce UV-induced damage/degradation of plastics and packaged food [1]. Nevertheless, there is great interest in replacing UV-stabilized packaging products already available on the market with technologies based on bio-sourced, abundant and potentially non-toxic and biodegradable organic biopigments, to develop environmentally benign pigmentmatrix multi-materials for the packaging industry [2].

This work was supported in part by NSERC DG (CS) and FRQNT Team Grant"

Prof. Dr. Clara Santato (corresponding author) is at the Engineering Physics department of the university "Polytechnique Montréal", Succ. Centre-ville, Montréal, Québec, H3C 3A7, Canada (e-mail: clara.santato@polymtl.ca).

Prof. Dr. Fabio Cicoira at the Chemical Engineering department of the university "Polytechnique Montréal", Succ. Centre-ville, Montréal, Québec, H3C 3A7, Canada (e-mail: fabio.cicoira@polymtl.ca).

Prof. Dr. Christian Pellerin is at the Chemistry department of "Universite de Montréal”, C.P. 6128, Succ. Centre-Ville, Montréal, QC, H3C 3J7, Canada (email: c.pellerin@umontreal.ca).
Nanoscience and nanotechnology are key resources for the exploration and exploitation of the full potential of organic biosourced materials for the packaging industry. Structure/process/property relationships have indeed to be established in this class of materials often featuring chemical and physical disorder, at the molecular and supramolecular scale [3]. For instance, the dimensionality of an organic biopigment (i.e. its monomer, oligomer, polymer nature) affects the absorption of the final packaging multi-material (with monomers and short oligomers expected to exhibit a blueshifted absorption with respect to polymers with extended electronic conjugation) [4].

Melanin is a pigment ubiquitous in flora and fauna [5]. In humans, various forms of melanin are responsible for a variety of functions: photoprotection of skin and eyes (brown-black eumelanin), hair and eye color (eumelanin and yellow-reddish pheomelanin), neurotransmission in the brain substantia nigra (neuromelanin) [6]. The ink of Sepia officinalis, used by squids for camouflage or defense, contains Sepia Melanin (natural eumelanin) [7]. Eumelanin, the form of melanin most investigated by materials scientists, henceforth indicated as "melanin", is a chemically heterogeneous biomacromolecule, consisting of randomly cross-linked oligomers of the 5,6dihydroxyindole (DHI) and DHI-2-carboxylic acid (DHICA) building blocks. Melanin features broadband UV-Vis absorption, metal chelation as well as anti-oxidant and radical scavenging properties [8]. In addition, it is non-toxic [9] and biodegradable [10], which are of paramount importance for food-packaging industry.

Different types of eumelanin (Sepia Melanin, fungal melanin and polydopamine, a eumelanin-like synthetic material) [11] have been evaluated as additives to enhance the absorption in the UV range of polymers such as poly(vinyl alcohol) (PVA) [12], polyimide (PI) [13] and poly(lactic acid) (PLA) [14].

Eduardo Di Mauro is at the Engineering Physics department of the university "Polytechnique Montréal", Succ. Centre-ville, Montréal, Québec, H3C 3A7, Canada (e-mail: eduardo.di-mauro@polymtl.ca).

Dr. Xinda Li is at the Chemical Engineering department of the university "Polytechnique Montréal", Succ. Centre-ville, Montréal, Québec, H3C 3A7, Canada (e-mail: xinda.li@polymtl.ca). 
The role of melanin regarding the polymers' photostability (stability under prolonged UV irradiation) is still under debate: melanin has been reported to be a photo-stabilizer (i.e. limiting photo-oxidation) in some works dealing with PVA [15] and bisphenol A polycarbonate (PC) [16] while in another work regarding the ethylene-vinyl acetate copolymer (EVA) it has been reported to be a pro-oxidant (i.e. favoring photooxidation) [17]. Eumelanin has been also evaluated as a biosourced thermal stabilizer for PI [13], PVA [18], polyhydroxybutyrate (PHB) [19], poly(methyl methacrylate) (PMMA) [20], polypropylene (PP) [20] and for a bio-sourced polymer, carrageenan, extracted from red seaweeds [21], as it can scavenge the radicals formed during the thermal degradation of the polymers [1], [22].

In this work, we report on the addition of the biopigment eumelanin as UV stabilizer in commercial linear low-density polyethylene (LLDPE) matrices. We used eumelanin in the Melanin Free Acid form to improve its dispersion in the polymer matrix and reduce its visible absorption, and fabricated eumelanin-including films by melt-compounding and compression molding. The UV-visible absorption of the eumelanin-including films was characterized by UV-Vis spectroscopy. To evaluate the effects of long-term UV irradiation on the eumelanin-including films, a UV-aging test was carried out.

\section{MATERIALS AND METHODS}

A commercial grade of LLDPE, namely LL6407.67 from Exxon Mobil ${ }^{\mathrm{TM}}$ (including additives undisclosed by the company that we attempted to identify using LC/UV/MS chromatography, i.e. high-performance liquid chromatography coupled to UV spectrum detection and mass spectrometry), was investigated in this work (monomer in Fig. S1-a). Its melting temperature is $127^{\circ} \mathrm{C}$.

The pellets as received from the company were firstly used to fabricate films of pure LL6407.67. The fabrication implied melting the pellets in a batch mixer (Brabender Plasticorder DDRV501) and then compression-molding in a hot press. Stabilization in in a cold press ensued (processing parameters in Tables S1-S4). The size of the so-obtained films was $20.1 \mathrm{~cm}$ $\times 15.0 \mathrm{~cm}$. Such films will be from now on referred to as "control films".

This study entailed three types of eumelanin, one bio-sourced and two synthetic. Bio-sourced eumelanin was extracted from cuttlefish ink (Sepia Melanin), following procedures established in the literature [17]. The two synthetic eumelanins were synthesized from only one of the two monomers (DHI or DHICA), i.e. DHI-melanin and DHICA-melanin, by a solidstate polymerization method [24]. Eumelanin was included in the polymers by melt compounding in the batch mixer. The eumelanin-including films featured the same processing parameters as the control films (Tables S1-S4).

The Melanin Free Acid (MFA) treatment of eumelanin was deemed necessary before the addition of eumelanin to the plastics to properly distribute eumelanin in the commercial LLDPE during melt compounding in the batch mixer [17], [25]. Eumelanin was added to the plastics in the amount $0.2 \%$ wt. In the case of synthetic DHICA-melanin, higher amounts $(0.5 \%$ wt. and $0.8 \%$ wt.) were investigated because DHICA-melanin has a more intense absorption in the UV region and a lower visible absorption with respect to the other two types (synthetic DHI-melanin and natural Sepia Melanin) [17], [26].

A commercial UV-absorber, 2-hydroxy-4(octyloxy)benzophenone, also called BP12 [27] (commercial name BLS ${ }^{8} 531$ from Mayzo), was also considered (Fig. S1-b), for the sake of comparison.

A PerkinElmer LAMBDA 1050 spectrophotometer equipped with a Labsphere integrating sphere was used to measure the UV-visible absorption of the films. The samples tested (ca. 2 $\mathrm{cm} \times 3 \mathrm{~cm}$ ) were cut from the center of the compression-molded films and placed inside the sphere at a $20^{\circ}$ angle with respect to the incident radiation beam. This center-mount setup entailed the simultaneous measurement of the total reflected $R$ and transmitted $T$ radiation in the $250-800 \mathrm{~nm}$ range. The resulting absorption of the films was inferred by posing $A=1-(R+$ $T)$. An equivalent absorption coefficient $\alpha\left(\mathrm{cm}^{-1}\right)$ was inferred by assuming a Beer-Lambert-like attenuation, given that the films feature different thicknesses [17] (Fig. S2). The equivalent absorption coefficient of the commercial LLDPE including eumelanin or the commercial benzophenone will be referred to as $\alpha_{\text {ADD }}$ whereas the control films' equivalent absorption coefficient as $\alpha_{\mathrm{CON}}$. The relative increase of absorption provided by the additive in the films with respect to the control film is thus indicated by the ratio $\alpha_{A D D} / \alpha_{C O N}$.

Thermogravimetric analyses (TGAs) shed light over whether the addition of eumelanin changes the degradation temperature of the polymer towards lower or higher temperatures, due to antagonistic or synergistic effects with the anti-oxidant additives already present in the commercial LLDPE. A TA Instruments TGA 2950 thermogravimetric analyzer was used for the thermogravimetric analyses, carried out in argon and air atmosphere $\left(90 \mathrm{~cm}^{3} \mathrm{~min}^{-1}\right)$, at a heating rate of $10^{\circ} \mathrm{C} \mathrm{min}^{-1}$, in the range of $25-600{ }^{\circ} \mathrm{C}$. Following ASTM E 2550-07, the onset degradation temperature, $\mathrm{T}_{\text {on }}$, was calculated, setting $0.005 \% /{ }^{\circ} \mathrm{C}$ as the value for which the initial plateau of the derivative of the TGA (DTG) was considered over. The temperature of the maximum rate of degradation is also reported. For the average values, 3 samples for the control films and 2 samples for all the other cases were considered.

A UV-aging test entailing prolonged UV-exposure was performed to evaluate whether the absorption enhancement provided by eumelanin (MFA) prevents or favors photodegradation. The lamp used featured an intensity of $\sim 10$ $\mathrm{W} \cdot \mathrm{m}^{-2}$ in the $290 \mathrm{~nm}-440 \mathrm{~nm}$ spectral range and the films were held at a $10 \mathrm{~cm}$-distance from the lamp. Such a test lasted 144 days, in air, at $25^{\circ} \mathrm{C}$.

The possible presence of photodegradation products was assessed at day 48 and day 144 of UV irradiation, by means of infrared spectroscopy (IR), UV-visible spectroscopy and thermogravimetric analyses. Optical images of the samples 
were taken at days $0,48,96$ and 144 of UV irradiation (Table S4).

The attenuated total reflection (ATR) mode was used to record the IR spectra of the films, using a Bruker Optics Tensor 27 FTIR spectrometer equipped with a HgCdTe detector. Samples were pressed against the silicon ATR element of a MIRacle accessory (Pike Technologies). The resolution was $4 \mathrm{~cm}^{-1}$ and the spectra were obtained by averaging at least 250 scans. The maximum value of absorbance of each spectrum was taken as a reference for normalization.

\section{RESULTS}

The equivalent absorption coefficient $\left(\mathrm{cm}^{-1}\right)$ of the control film $\left(\alpha_{\mathrm{CON}}\right)$ was compared with that of films including eumelanin $\left(\alpha_{A D D}\right)$.

The peak located at approximately $270 \mathrm{~nm}$ with a shoulder in the interval $300-340 \mathrm{~nm}$ (likely attributable to additives in the commercial grade polymer matrix) may be ascribed to the presence of tris(2,4-di-t-butylphenyl) phosphite, an antioxidant commonly present in commercial grade LLDPEs [28]. In films containing the three types of eumelanin, an absorption peak located at ca. $315 \mathrm{~nm}$ is observable (Fig. 1, a-b): it most likely results from the overlap of the absorption of collections of oligomers of DHI and DHICA [29]. Polypyrroles resulting from the peroxidation of eumelanin during the MFA treatment could provide a further contribution [30], [31]. The amount of eumelanin $0.2 \%$ wt., both for synthetic and natural eumelanin, brings about an absorption increase in the UVA and visible ranges. While in the case of Sepia Melanin $\alpha_{A D D} / \alpha_{C O N}$ reaches values as high as 40 in the visible range, at $590 \mathrm{~nm}$, for the synthetic counterparts, it attains a maximum value of 12 to 13 at the same wavelength. In general, for applications where color must not to be altered, a negligible absorption increase is desirable in the visible range ( ${ }^{\alpha_{A D D}} / \alpha_{C O N}$ close to 1$)$ : it can be then inferred that DHICA-melanin and DHI-melanin perform better than Sepia Melanin in that context. However, if a black color is desired, Sepia Melanin may represent a bio-sourced alternative to UV-absorbers based on petroleum (e.g. carbon black [32]).

The MFA treatment was carried out on the three types of eumelanin used in this work prior to addition to the commercial grade LLDPE. Such a treatment brings about the de-stacking of eumelanin's supramolecular structure, as eumelanin aggregates dissociate into smaller protomolecules [25]. The oligomers' length is also reduced by the treatment [25]. Both the destacking and the reduced oligomers size reduce the electronic delocalization; consequently, the absorption in the visible range is reduced [23]. The weaker optical absorption in the visible region provided to commercial LLDPE by the synthetic eumelanins with respect to Sepia Melanin may be ascribed to the less complex initial supramolecular assembly of the synthetic eumelanins, which was more easily "opened" by the MFA treatment [33].

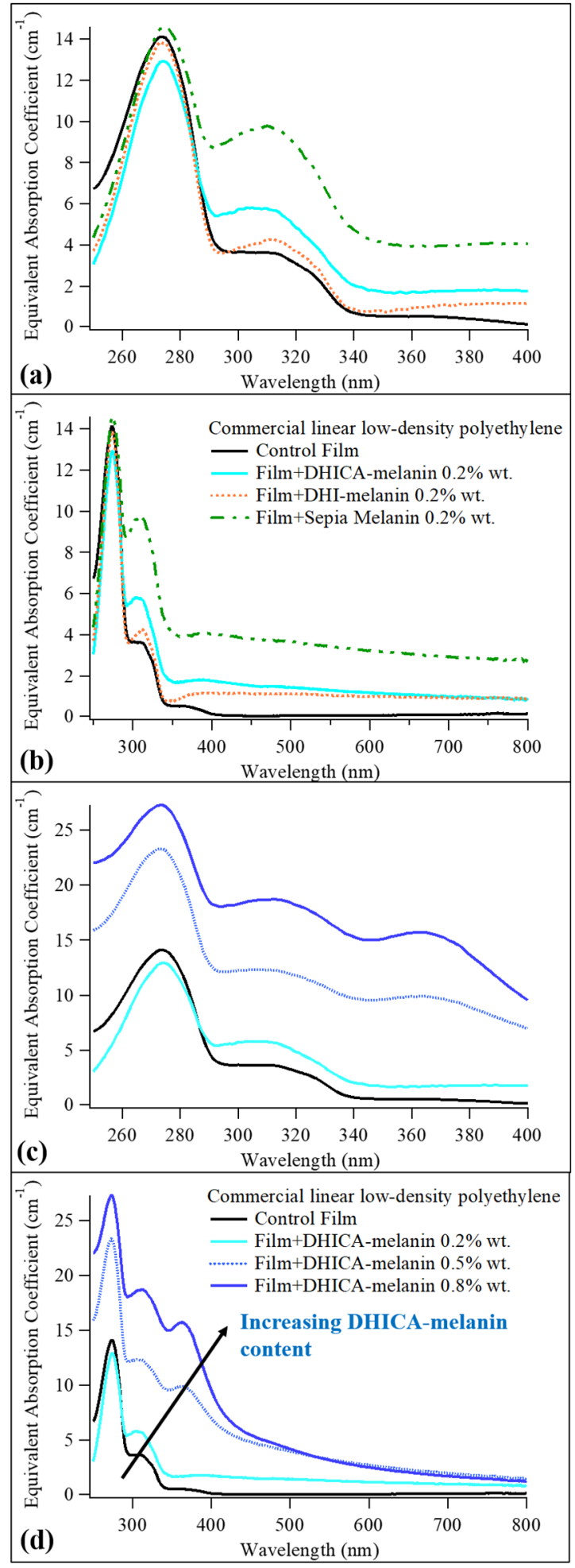

Fig. 1 Equivalent absorption coefficient of the control films and films containing different types of eumelanin (a-b) and increasing amounts of DHICA-melanin (c-d): in the UV range $(a, c)$ and in the UV and visible ranges $(b, d)$.

The darker color of the pellets and films including Sepia Melanin with respect to its synthetic counterparts confirm this hypothesis (Table S3). As pointed out in a previous work of our research group [17], adding an as-polymerized synthetic 
powder (DHICA-melanin and DHI-melanin) or as-extracted bio-sourced powder (natural Sepia Melanin), without the MFA treatment, to the batch mixer, brought about films with black dots and poor additive dispersion after compression molding. DHICA-melanin has lower visible absorption and more intense absorption in the UV region [34] with respect to the other two types of eumelanin (DHI-melanin and Sepia Melanin), so concentrations of DHICA-melanin higher than $0.2 \%$ wt. were also tested $(0.5 \%$ wt. and $0.8 \%$ wt.). With increasing DHICAmelanin content, a further absorption peak, at ca. $370 \mathrm{~nm}$, is observable: it can be assigned to a collection of oligomers, too (Fig. 1, c-d) [29], [35]. The equivalent absorption coefficient increases with increasing DHICA-melanin amount more remarkably in the UV range than in the visible range (Fig. S2): in other words, increasing the eumelanin content from $0.2 \% \mathrm{wt}$. to $0.8 \%$ wt. benefits the UV range absorption more than the visible range absorption.

Regarding the thermal degradation, for LLDPE, in inert atmosphere, the main step (massive cleavage of polymer chains) takes place at $\sim 470{ }^{\circ} \mathrm{C}[36]$ (Fig. S3). In air, the thermooxidative degradation is faster [37]: it starts with an initial gain in mass, ascribed to an oxygen uptake by the polyethylene fraction, bringing about the formation of alkyl-hydroperoxides [38]. This is followed by a mass drop due to thermo-oxidative cleavage of the backbone chains (Fig. S4) [38].

The addition of eumelanin to the commercial LLDPE leaves both the onset degradation temperature and the main degradation peak unaltered (Fig. S3-S4). Eumelanin, in the MFA form, did not show any antagonistic nor synergistic effects with the anti-oxidant most likely already present (tris(2,4-di-t-butylphenyl) phosphite), as our results show that it does not interfere with the thermo-oxidative stabilization imparted by such a stabilizer. In the literature, it has been claimed that the biopigment is able to provide thermal stabilization (higher degradation temperatures) to polymers, in virtue of the radical scavenging properties of eumelanin [13], [18]-[20], [39]. It can thus be hypothesized that the radical scavenging ability of eumelanin has been reduced by the MFA treatment; such a decrease due to the MFA treatment has already been reported in the literature [17], [40].

We then evaluated the long-term effects of the UV-absorption enhancement imparted to our commercial LLDPE through a UV-aging test. As a matter of fact, the broad UV-absorption of eumelanin confers to the biopigment a double role, either photoprotective or photodamaging [41]. The main photoprotection path entails the conversion by eumelanin of the photons' energy into heat (photothermal effect) [42]. UVabsorbers protect polymers against photo-oxidation by the same effect [43]. The quenching of reactive oxygen species (ROS) generated upon UVA irradiation [44] represents another photoprotection path enabled by eumelanin. Notwithstanding, eumelanin's photodegradation, with fission of the indolequinone moiety, can occur under prolonged exposure to UVA [45]. The loss of its radical scavenging abilities can result from this photo-induced chemical modification [46], with eumelanin becoming a pro-oxidant [47].

IR spectroscopy was used to chemically identify possible photodegradation products in the polymer films during the UVaging test. After 48 days of UV-aging, the control film showed no substantial photodegradation (Fig. S5). As opposed to that, the IR spectrum at the end of the UV irradiation (144 days) shows a band centered at $1715 \mathrm{~cm}^{-1}$ (carbonyl groups, the main photo-oxidation products) [36] (Fig. 2).

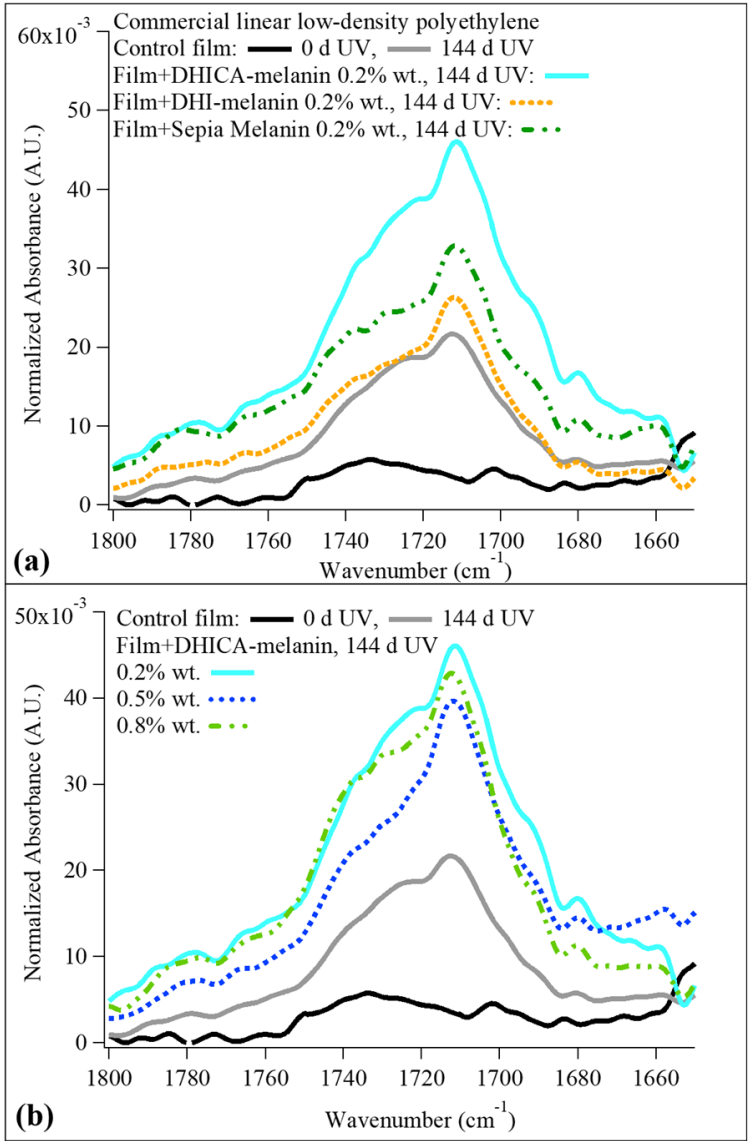

Fig. 2 IR spectra $\left(1800 \mathrm{~cm}^{-1}-1650 \mathrm{~cm}^{-1}\right)$ of the control film and films including eumelanin, at day 0 and 144 of UV-aging: (A) the 3 types of eumelanin $0.2 \%$ wt.; (B) different concentrations of DHICA-melanin. For all the films, the amount of eumelanin was too low to be detected in the IR spectra so that only the control film is shown at day 0 of UVaging.

In presence of eumelanin, after 48 days of UV irradiation, differently from the control film, a certain extent of photodegradation could be observed (band at $1715 \mathrm{~cm}^{-1}$ attributed to carbonyl groups [36]) (Fig. S5). After 144 days of UV-Aging, photo-oxidation became more pronounced: a further band of a photodegradation product, $\gamma$-lactone, at 1780 $\mathrm{cm}^{-1}$, is present [48] (Fig. 2). Photodegradation of the films including eumelanin occurred to a larger extent than for the control film of commercial LLDPE.

The control film presents a decrease of the equivalent absorption coefficient, in the first 48 days of UV irradiation, at 
approximately $270 \mathrm{~nm}$ and $304 \mathrm{~nm}$, most likely due to the depletion of the anti-oxidants (such as tris(2,4-di-t-butylphenyl) phosphite) already present in the commercial grade polymer responsible for such peaks. Between day 48 and 144 of UV exposure, the equivalent absorption coefficient increases in the UVC range, due to the absorption of photodegradation products [49] (Fig. 3 and Table S6).

The UV absorption enhancement imparted by eumelanin is only partially reduced after 48 days of UV irradiation: for example, in the case of the film including $0.8 \% \mathrm{wt}$. eumelanin, the equivalent absorption coefficient at the two absorption peaks characteristic of the biopigment, at ca. $315 \mathrm{~nm}$ and ca. $370 \mathrm{~nm}$, decreases by $10 \%$ and $20 \%$, respectively (Fig. 3 ). The decrease of the absorption peak at $315 \mathrm{~nm}$ can be related to the photodegradation of polypyrroles [50] whereas the reduction of the peak at ca. $370 \mathrm{~nm}$ (due to collections of oligomers [29], [35]) is related to the UVA-induced oxidative degradation of eumelanin, bringing to the formation of pyrrole-2,3,5tricarboxylic acid and fission of indolequinone [51].

Furthermore, the equivalent absorption coefficient of the films including eumelanin increases in the UVC range between day 0 and 48. Subsequently, between day 48 and 144 of UV-aging it increases over the entire UV range: this effect is related to the formation of photodegradation products (mainly carbonyl groups) that absorb in that wavelength range [49] (Fig. 3 and Table S6).

In order to explain the higher degree of photodegradation observed with the films containing eumelanin with respect to the control film (pro-oxidant behavior of eumelanin), it has to be taken into consideration that, during prolonged UVA irradiation of eumelanin in solution, reactive oxygen species (ROS) are generated together with the characteristic photodegradation products of eumelanin, pyrrole-2,3,5tricarboxylic acid and fission of indolequinone [45], [46].

As we already suggested for another polyethylene based polymer [17], the ROS formation may take place also when eumelanin is embedded in the polymer matrix under prolonged UVA irradiation. As eumelanin is in the MFA form, with decreased radical scavenging ability, ROS cannot be efficiently quenched by eumelanin, thus contributing to the photooxidation process of the commercial LLDPE [52].

The thermal decomposition of such degradation products may explain why the onset degradation temperature $\left(T_{o n}\right)$ of the samples including eumelanin decreases as a result of the UVaging treatment to a higher extent with respect to the control film both in inert atmosphere and in air [53] (Fig. S6-S7). Mechanical embrittlement of the films, which is a wellestablished consequence of photo-oxidation [54] took place (Table S4).

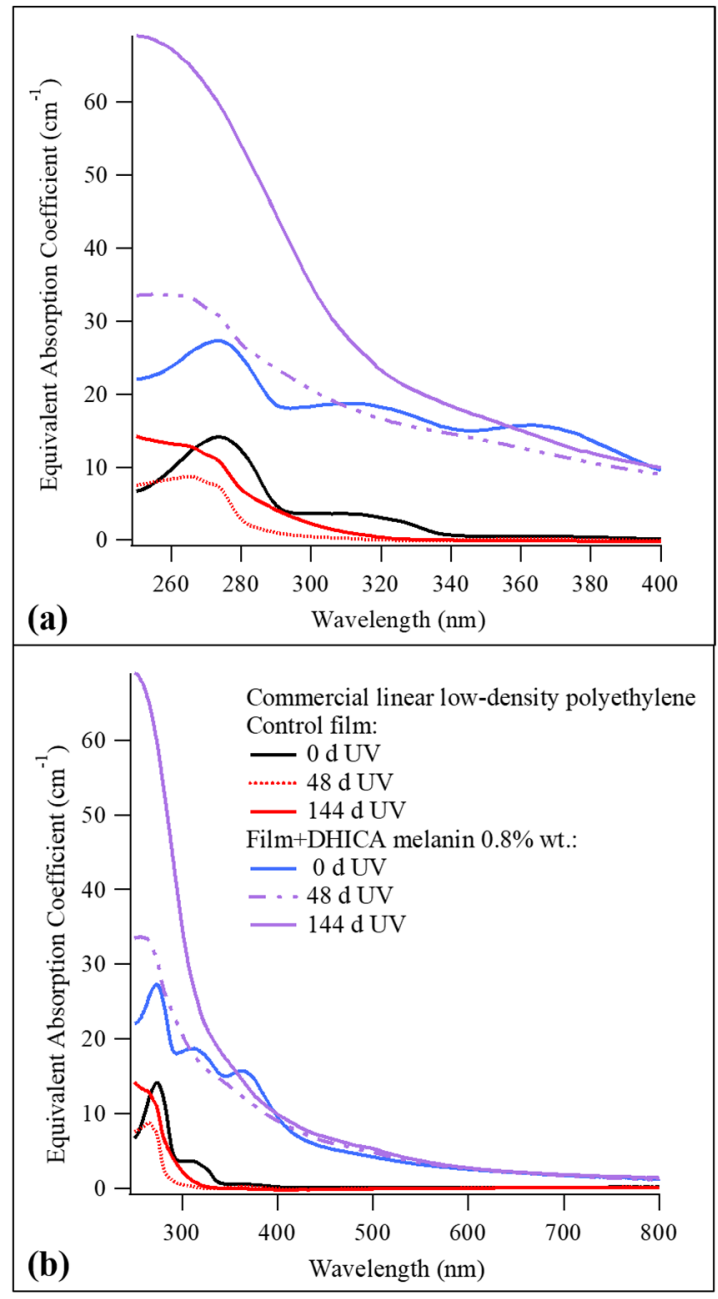

Fig. 3 Equivalent absorption coefficient (a) in the UV range and (b) in the UV and visible ranges for the control film and the film containing DHICA-melanin $0.8 \%$ wt. at different times of UVaging $(0,48$ and 144 days).

The commercial benzophenone BLS $₫ 531$ provides a one-totwo order of magnitude increase of the absorption coefficient in the UV range with a sharp absorption drop between the UV and the visible ranges (Fig. S8). BLS®531 limited, but did not completely avoid, the formation of photodegradation products after 144 days of UV-aging (Fig. S9). The UV-aging treatment consumes such an additive, similarly to the UV-absorption enhancement due to eumelanin: the absorption coefficient indeed shifts towards lower values in the UV-range as the UVaging time elapses (decrease of 10\% - 30\% after 48 days, and of $60 \%-70 \%$ after 144 days) (Fig. S8).

\section{CONCLUSIONS}

In summary, the addition of eumelanin, both synthetic and natural, in the amount $0.2 \%$ wt., to a commercial grade LLDPE, caused an increase of the absorption mainly in the UVA and visible regions. Increasing the amount to $0.8 \%$ wt. for DHICAmelanin (synthetic eumelanin obtained from only one of the two building blocks of eumelanin, DHICA) provides a substantial UV-absorption increase that was almost entirely kept after prolonged exposure to UV. The Melanin Free-Acid (MFA) 
treatment, which was necessary to disperse the biopigment in the polymer matrix, most likely brought about the pro-oxidant (i.e. photodegradation favoring) role of eumelanin during prolonged UV irradiation. Strategies other than the MFA treatment will have to be adopted to ensure the proper mixing between the polymer matrix and the melanin. Work is in progress to control the molecular and supramolecular structure of eumelanin by engineering the solid-state polymerization process (e.g. controlling the $\mathrm{O}_{2}$ concentration and relative humidity in the atmosphere during the polymerization as well as the time of polymerization), to obtain eumelanin nanostructures of controlled size prior to their mixing with the polymer matrix. A further benefit of this approach would be the possibility to tailor the UV-Vis absorption properties of eumelanin nanostructures.

\section{ACKNOWLEDGMENT}

The authors thank R. Silverwood and prof. A. Ajji for the help with polymer processing, Dr. A. Pezzella for help with the Melanin Free Acid treatment, Dr. B. Baloukas for the help with the UV-visible characterization, Dr. A. Furtos for help with LC/UV/MS chromatography as well as C. Bayard, N. Vandooren, M. Camaggi and J. De Angelis for help with polymer processing and characterization.

\section{REFERENCES}

[1] S. E. M. Selke and J. D. Culter, Plastics Packaging: Properties, Processing, Applications, and Regulations, 3rd ed. Hanser Publications, Cincinnati, 2016.

[2] J. L. Koontz, J. E. Marcy, S. F. O'Keefe, S. E. Duncan, T. E. Long, and R. D. Moffitt, "Polymer processing and characterization of LLDPE films loaded with $\alpha$-tocopherol, quercetin, and their cyclodextrin inclusion complexes," J. Appl. Polym. Sci., vol. 117, no. 4, pp. 2299-2309, Aug. 2010.

[3] A. Antidormi, C. Melis, E. Canadell, and L. Colombo, "Understanding the Polymerization Process of Eumelanin by Computer Simulations," J. Phys. Chem. $C$, vol. 122, no. 49, pp. 28368-28374, 2018.

[4] H. Eckhardt, L. W. Shacklette, K. Y. Jen, and R. L. Elsenbaumer, "The electronic and electrochemical properties of poly(phenylene vinylenes) and poly(thienylene vinylenes): An experimental and theoretical study," J. Chem. Phys., vol. 91, no. 2, pp. 1303-1315, 1989.

[5] M. d'Ischia et al., "Melanins and melanogenesis: Methods, standards, protocols," Pigment Cell Melanoma Res., vol. 26, no. 5, pp. 616-633, 2013.

[6] M. d'Ischia et al., "Melanins and melanogenesis: from pigment cells to human health and technological applications," Pigment Cell Melanoma Res., vol. 28, no. 5, pp. 520-544, Sep. 2015.

[7] R. Xu, C. T. Prontera, E. Di Mauro, A. Pezzella, F. Soavi, and C. Santato, "An electrochemical study of natural and chemically controlled eumelanin," $A P L$ Mater., vol. 5, no. 12, p. 126108, Dec. 2017.

[8] P. Meredith and T. Sarna, "The physical and chemical properties of eumelanin," Pigment Cell Res., vol. 19, no. 6, pp. 572-594, Dec. 2006.

[9] M. Araújo et al., "Natural melanin: A potential pHresponsive drug release device," Int. J. Pharm., vol. 469, no. 1, pp. 140-145, Jul. 2014.

[10] E. Di Mauro, D. Rho, and C. Santato, "Biodegradation of Bio-Sourced and Synthetic Organic Electronic Materials: Towards Green Organic Electronics," Submitted.

[11] F. Deng et al., "The effect of dopamine modified titanium dioxide nanoparticles on the performance of Poly (vinyl alcohol)/titanium dioxide composites," Compos. Sci. Technol., 2017.

[12] Y. Wang et al., "A Novel UV-Shielding and Transparent Polymer Film: When Bioinspired Dopamine-Melanin Hollow Nanoparticles Join Polymers," ACS Appl. Mater. Interfaces, vol. 9, no. 41, pp. 36281-36289, Oct. 2017.

[13] Q. Li, G. Liao, J. Tian, and Z. Xu, "Preparation of Novel Fluorinated Copolyimide/Amine-

Functionalized Sepia Eumelanin Nanocomposites with Enhanced Mechanical, Thermal, and UV-Shielding Properties," Macromol. Mater. Eng., vol. 1700407, p. 1700407, 2017.

[14] Ł. Łopusiewicz, F. Jedra, and M. Mizieińska, "New poly(lactic acid) active packaging composite films incorporated with fungal melanin," Polymers (Basel)., vol. 10, no. 4, pp. 5-8, 2018.

[15] Y. Wang et al., "Simultaneous Enhancements of UVShielding Properties and Photostability of Poly(vinyl alcohol) via Incorporation of Sepia Eumelanin," $A C S$ Sustain. Chem. Eng., vol. 4, no. 4, pp. 2252-2258, Apr. 2016.

[16] Y. Wang et al., "Effects of Melanin on Optical Behavior of Polymer: From Natural Pigment to Materials Applications," ACS Appl. Mater. Interfaces, vol. 10, no. 15, 2018.

[17] E. Di Mauro et al., "Eumelanin for nature-inspired UV-absorption enhancement of plastics," Polym. Int., vol. 68, no. 5, pp. 984-991, May 2019.

[18] J. Y. Cheon and W. H. Park, "Enhanced thermal stabilization of polymer nanofibrous web using selfpolymerized 3,4-dihydroxy-L-phenylalanine," Polymer (Guildf)., vol. 125, pp. 126-133, Sep. 2017.

[19] G. S. Kiran, S. A. Jackson, S. Priyadharsini, A. D. W. Dobson, and J. Selvin, "Synthesis of Nm-PHB (nanomelanin-polyhydroxy butyrate) nanocomposite film and its protective effect against biofilm-forming multi drug resistant Staphylococcus aureus," Sci. Rep., vol. 7, no. 1, p. 9167, Dec. 2017.

[20] K. Shanmuganathan, J. H. Cho, P. Iyer, S. Baranowitz, and C. J. Ellison, "Thermooxidative Stabilization of Polymers Using Natural and Synthetic Melanins," Macromolecules, vol. 44, no. 24, pp. 9499-9507, Dec. 2011.

[21] S. Roy and J.-W. Rhim, "Preparation of carrageenanbased functional nanocomposite films incorporated with melanin nanoparticles," Colloids Surfaces B Biointerfaces, vol. 176, no. September 2018, pp. 317324, 2019.

[22] P. Meredith and T. Sarna, "The physical and chemical 
properties of eumelanin," Pigment Cell Res., vol. 19, no. 6 , pp. 572-594, 2006.

[23] K. B. Stark et al., "Effect of stacking and redox state on optical absorption spectra of melanins -comparison of theoretical and experimental results.," J. Phys. Chem. B, vol. 109, pp. 1970-1977, 2005.

[24] A. Pezzella et al., "Stem cell-compatible eumelanin biointerface fabricated by chemically controlled solid state polymerization," Mater. Horiz., vol. 2, no. 2, pp. 212-220, 2015.

[25] K. C. Littrell, J. M. Gallas, G. W. Zajac, and P. Thiyagarajan, "Structural Studies of Bleached Melanin by Synchrotron Small-angle X-ray Scattering," Photochem. Photobiol., vol. 77, pp. 115-120, 2003.

[26] L. Panzella et al., "Red human hair pheomelanin is a potent pro-oxidant mediating UV-independent contributory mechanisms of melanomagenesis," Pigment Cell Melanoma Res., vol. 27, no. 2, pp. 244 252, 2014.

[27] Q. Liu, Z. Chen, D. Wei, and Y. Du, “Acute toxicity formation potential of benzophenone-type UV filters in chlorination disinfection process," J. Environ. Sci. (China), vol. 26, no. 2, pp. 440-447, 2014.

[28] N. Haider and S. Karlsson, "A rapid ultrasonic extraction technique to identify and quantify additives in poly (ethylene)," Analyst, vol. 168, no. 3, pp. 797800, 1999.

[29] M. D'Ischia et al., "Structural effects on the electronic absorption properties of 5,6-dihydroxyindole oligomers: The potential of an integrated experimental and DFT approach to model eumelanin optical properties," Photochem. Photobiol., vol. 84, no. 3, pp. 600-607, 2008.

[30] A. Napolitano, A. Pezzella, M. D'Ischia, and G. Prota, "New pyrrole acids by oxidative degradation of eumelanins with hydrogen peroxide. Further hints to the mechanism of pigment breakdown," Tetrahedron, vol. 52, no. 26, pp. 8775-8780, 1996.

[31] R. A. W. Smith et al., "Mechanistic insights into the bleaching of melanin by alkaline hydrogen peroxide," Free Radic. Biol. Med., vol. 108, no. November 2016, pp. 110-117, 2017.

[32] M. Arroyo, M. A. López-Manchado, and B. Herrero, "Organo-montmorillonite as substitute of carbon black in natural rubber compounds," Polymer (Guildf)., vol. 44, no. 8, pp. 2447-2453, 2003.

[33] C. M. R. Clancy and J. D. Simon, "Ultrastructural organization of eumelanin from Sepia officinalis measured by atomic force microscopy," Biochemistry, vol. 40, no. 44, pp. 13353-13360, 2001.

[34] L. Panzella et al., "Atypical structural and $\pi$-electron features of a melanin polymer that lead to superior free-radical-scavenging properties," Angew. Chemie Int. Ed., vol. 52, no. 48, pp. 12684-12687, 2013.

[35] A. Pezzella et al., "Lack of visible chromophore development in the pulse radiolysis oxidation of 5,6dihydroxyindole-2-carboxylic acid oligomers: DFT investigation and implications for eumelanin absorption properties," J. Org. Chem., vol. 74, no. 10, pp. 3727-3734, 2009.
[36] T. Corrales, F. Catalina, C. Peinado, N. S. Allen, and E. Fontan, "Photooxidative and thermal degradation of polyethylenes: interrelationship by chemiluminescence, thermal gravimetric analysis and FTIR data," J. Photochem. Photobiol. A Chem., vol. 147, no. 3, pp. 213-224, Apr. 2002.

[37] V. Ambrogi et al., "An antioxidant bioinspired phenolic polymer for efficient stabilization of polyethylene," Biomacromolecules, vol. 15, no. 1, pp. 302-310, 2014.

[38] P. Persico, V. Ambrogi, C. Carfagna, P. Cerruti, I. Ferrocino, and G. Mauriello, "Nanocomposite polymer films containing carvacrol for antimicrobial active packaging," Polym. Eng. Sci., vol. 49, no. 7, pp. 1447-1455, Jul. 2009.

[39] W. Dong et al., "Enhanced thermal stability of poly(vinyl alcohol) in presence of melanin," J. Therm. Anal. Calorim., vol. 115, no. 2, pp. 1661-1668, Feb. 2014.

[40] E. Kim et al., "Spectroelectrochemical Reverse Engineering DemonstratesThat Melanin's Redox and Radical Scavenging Activities Are Linked," Biomacromolecules, p. acs.biomac.7b01166, 2017.

[41] F. Solano, "Photoprotection versus photodamage: updating an old but still unsolved controversy about melanin," Polym. Int., vol. 65, no. 11, pp. 1276-1287, Nov. 2016.

[42] S. E. Forest and J. D. Simon, "Wavelength-dependent Photoacoustic Calorimetry Study of Melanin," Photochem. Photobiol., vol. 68, no. 3, pp. 296-298, 1998.

[43] P. Gijsman, "Polymer Stabilization," in Applied Plastics Engineering Handbook, Elsevier, 2017, pp. 395-421.

[44] S. Ito, L. Kolbe, G. Weets, and K. Wakamatsu, "Visible light accelerates the ultraviolet A-induced degradation of eumelanin and pheomelanin," Pigment Cell Melanoma Res., p. pcmr.12754, Dec. 2018.

[45] G. Szewczyk, A. Zadlo, M. Sarna, S. Ito, K.

Wakamatsu, and T. Sarna, "Aerobic photoreactivity of synthetic eumelanins and pheomelanins: generation of singlet oxygen and superoxide anion," Pigment Cell Melanoma Res., Aug. 2016.

[46] S. Ito, K. Wakamatsu, and T. Sarna, "Photodegradation of Eumelanin and Pheomelanin and Its Pathophysiological Implications," Photochem. Photobiol., vol. 94, no. 3, pp. 409-420, 2018.

[47] T. Sarna, "Properties and function of the ocular melanin - A photobiophysical view," J. Photochem. Photobiol. B Biol., vol. 12, no. 3, pp. 215-258, Feb. 1992.

[48] J. V Gulmine, P. R. Janissek, H. M. Heise, and L. Akcelrud, "Degradation profile of polyethylene after artificial accelerated weathering," Polym. Degrad. Stab., vol. 79, pp. 385-397, 2003.

[49] A. Tidjani and C. A. Wilkie, "Photo-oxidation of polymeric-inorganic nanocomposites: chemical, thermal stability and fire retardancy investigations," Polym. Degrad. Stab., vol. 74, no. 1, pp. 33-37, Jan. 2001. 
[50] O. Inganäs, T. Skotheim, and I. Lundström,

"Polypyrrole-semiconductor Schottky barriers," J. Appl. Phys., vol. 54, no. 6, pp. 3636-3639, 1983.

[51] K. Wakamatsu, Y. Nakanishi, N. Miyazaki, L. Kolbe, and S. Ito, "UVA-induced oxidative degradation of melanins: Fission of indole moiety in eumelanin and conversion to benzothiazole moiety in pheomelanin," Pigment Cell Melanoma Res., vol. 25, no. 4, pp. 434445, 2012.

[52] J. W. Nicholson, "Polymer Degradation," in Chemistry of Polymers, 3rd ed., Royal Society of Chemistry, 2006.

[53] S. Ray and R. P. Cooney, "Thermal Degradation of Polymer and Polymer Composites," in Handbook of Environmental Degradation of Materials, Elsevier, 2012, pp. 213-242.

[54] E. Gauthier, B. Laycock, F. J. J. M. Cuoq, P. J. Halley, and K. A. George, "Correlation between chain microstructural changes and embrittlement of LLDPEbased films during photo- and thermo-oxidative degradation," Polym. Degrad. Stab., vol. 98, no. 1, pp. 425-435, 2013.

\section{TOC Graphical Abstract}

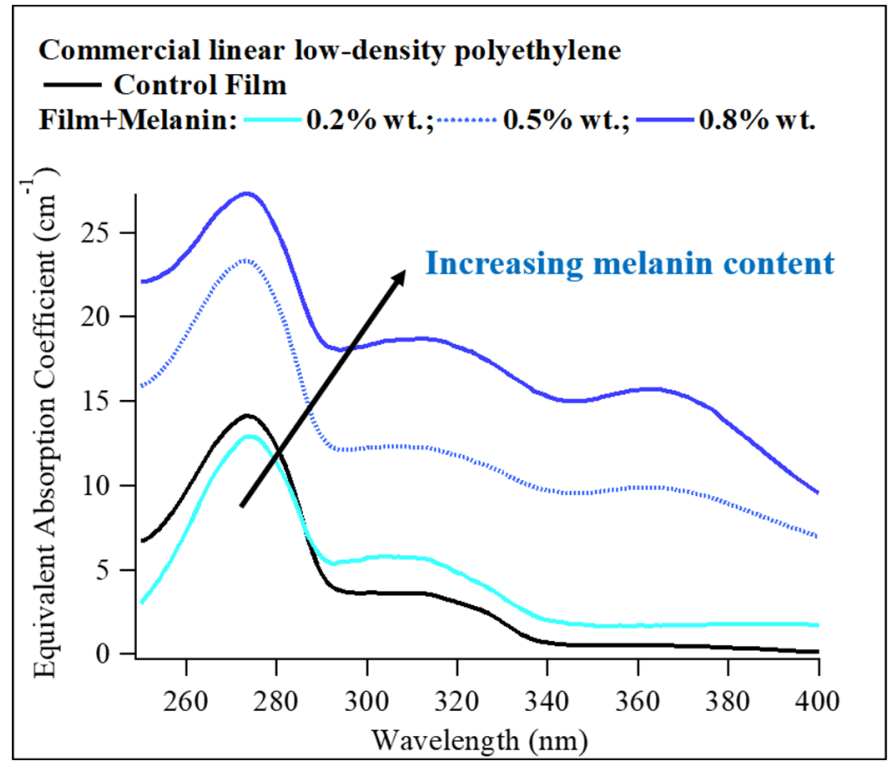

